\title{
Influence of microRNA-34a on proliferation, invasion and metastasis of HCT116 cells
}

\author{
CHUNYAN LI ${ }^{1}$, SHUMING LU ${ }^{1}$, YINGYAN WANG ${ }^{2}$, SHIBIN GUO $^{1}$, \\ TIANYU ZHAO ${ }^{1}$, XIAOSHENG WANG ${ }^{1}$ and BO SONG $^{3}$ \\ ${ }^{1}$ Department of Gastroenterology, The First Affiliated Hospital of Dalian Medical University, \\ Dalian, Liaoning 116011; ${ }^{2}$ Laboratory Center for Diagnostics and ${ }^{3}$ Department of Pathology, \\ Dalian Medical University, Dalian, Liaoning 116044, P.R. China
}

Received November 4, 2015; Accepted November 3, 2016

DOI: $10.3892 / \mathrm{mmr} .2016 .6088$

\begin{abstract}
The aim of the present study was to investigate the role of microRNA (miR)-34a expression in the proliferation, invasion and metastasis of colon cancer and its underlying mechanisms. HCT116 cells were cultured in high-sugar Dulbecco's modified Eagle's medium (DMEM) containing $10 \%$ fetal bovine serum and $1000 \mathrm{U} / \mathrm{ml}$ penicillin-streptomycin. Following digestion and resuspension, the cells were used for transfection, expression and western blot analysis. HCT116 cells from miR-34a transfection, negative control and blank control groups were seeded into a 96-well plate at a density of $10^{5} \mathrm{cells} / \mathrm{ml}$, and $200 \mu \mathrm{l}$ complete DMEM was added. The data are presented as the mean \pm standard error. A one-way analysis of variance was performed to compare groups. miR-34a-HCT116 cells demonstrated significantly increased expression levels of miR-34a. The proliferation of HCT116 cells with overexpression of miR-34a was significantly inhibited to $0.49 \pm 0.11$ compared with the blank control group $(\mathrm{P}<0.001)$. Compared with the blank control and negative control groups, the protein expression levels of B-cell lymphoma 2 (Bcl-2) were markedly reduced in the miR-34a transfected group. Furthermore, the protein expression levels of Bcl-2-associated X protein were significantly increased and those of matrix metalloproteinase (MMP)-2 and MMP-9 were markedly reduced in the miR-34a transfected group, MMP-9 to a greater extent. The present study suggested that
\end{abstract}

Correspondence to: Dr Chunyan Li, Department of Gastroenterology, The First Affiliated Hospital of Dalian Medical University, 222 Zhongshan Road, Dalian, Liaoning 116011, P.R. China

E-mail: chunyanlidl@163.com

Dr Bo Song, Department of Pathology, Dalian Medical University, 9th West Section, Lvshun South Road, Dalian, Liaoning 116044, P.R. China

E-mail: bo_song2014@163.com

Key words: microRNA-34a, HCT116 cells, colon cancer overexpression of miR-34a may inhibit the proliferation, invasion and metastasis of HCT116 cells.

\section{Introduction}

Colon cancer is one of the most common types of gastrointestinal cancers in China. It has the third greatest incidence of all cancer types. Epidemiological studies of the etiology and underlying mechanisms of colon cancer have demonstrated significant value for its early diagnosis, clinical treatment and prognosis. Tumor invasion and lymph node metastasis are important factors for determining the prognosis of the disease. At present, the primary treatment for colon cancer is chemotherapy. However, there are a number of severe adverse effects associated with currently available anticancer drugs, particularly chemotherapeutic agents, including nausea, vomiting, mouth ulcers, bone marrow suppression and hair loss. In addition, anticancer drugs may affect the heart, kidney, liver, lungs, as well as additional organs, which may lead to infertility or malformation. Furthermore, resistance to therapy is a major obstacle in the successful treatment of patients with colon cancer $(1,2)$. Therefore, novel individualized treatment strategies are required to improve the survival of patients, and the prevent cancer invasion and metastasis (3).

MicroRNA (miRNA) is a type of endogenous non-coding RNA with an evolutionarily highly conserved structure. Previous research involving miRNAs have revealed a role of miRNAs in the regulation of numerous cellular functions, including development and differentiation, cell cycle regulation, metabolism and apoptosis $(4,5)$. A large number of miRNAs are encoded by genes located in regions frequently exposed to changes in cancer cells, and alterations in miRNA expression levels have been associated with various cancers $(6,7)$. In addition, specific miRNA expression signatures have been demonstrated to correlate with the prognosis and progression of different cancers (8). By downregulating protein-encoding genes that promote or inhibit cell proliferation, several miRNAs have been demonstrated to function as tumor suppressors or oncogenes (6-8). miRNA (miR)-34a may be an effector molecule of the tumor suppressor gene $\mathrm{p} 53$, and thus inhibit the proliferation of various cancer cells (9-11). However, the underlying mechanisms of 
miR-34a and its role in colon cancer remain unclear. The present study investigated the role of miR-34a expression in the proliferation, invasion and metastasis of colon cancer and its underlying mechanisms, via construction of an miR-34a expression vector and its transfection into HCT116 colon cancer cells.

\section{Materials and methods}

Cell strains and reagents. The HCT116 human colon cancer cell line was purchased from the Cell Bank of the Chinese Academy of Sciences (Beijing, China). Cell culture reagents including high-sugar Dulbecco's modified Eagle's medium (DMEM), fetal bovine serum, penicillin and streptomycin were purchased from Gibco; Thermo Fisher Scientific, Inc. (Waltham, MA, USA), the miRNA Extraction kit was purchased from Qiagen, Inc. (Valencia, CA, USA), and the Taqman MicroRNA Reverse Transcription and the TaqMan MicroRNA Detection kit were obtained from Takara Biotechnology, Co., Ltd. (Dalian, China). MTT reagent was purchased from Sigma-Aldrich; Merck Millipore (Darmstadt, Germany), the miR-34a eukaryotic expression vector (GAU GUUCUAUUGAAGAGUGUUUG), miR-34a negative control (GGACACGAAATCTATGCGCGTG) and green fluorescent protein (GFP)-carrying recombinant plasmid were designed and synthesized by Shanghai GenePharma Co., Ltd. (Shanghai, China), and the transfection reagent Lipofectamine ${ }^{\circledR} 2000$ was obtained from Invitrogen; Thermo Fisher Scientific, Inc. Western blotting reagents (loading buffer, protein marker, electrophoretic liquid, transfer buffer and developer) were purchased from Beyotime Institute of Biotechnology (Haimen, China), and primary and secondary antibodies were purchased from Cell Signaling Technology, Inc. (Danvers, MA, USA). Other common reagents were purchased from Sigma-Aldrich; Merck Millipore.

Cell culture. HCT116 cells were cultured in high-sugar DMEM containing $10 \%$ fetal bovine serum and 1,000 U/ml penicillin-streptomycin, in a constant temperature incubator at $37^{\circ} \mathrm{C}$ and $5 \% \mathrm{CO}_{2}$. The medium was refreshed every 2-3 days. Trypsin was used to digest cells when they had reached $>80 \%$ confluence, before they were used for downstream experiments.

miR-34a transfection. Following digestion and resuspension, the HCT116 cells were diluted to $10^{5}$ cells $/ \mathrm{ml}$ with complete DMEM. Cells $(2 \mathrm{ml})$ were seeded into 6 -well plates and cultured in a constant temperature incubator at $37^{\circ} \mathrm{C}$ and $5 \%$ $\mathrm{CO}_{2}$ for $24 \mathrm{~h}$. The serum-free medium was replaced when cell fusion was $>50 \%$ and transfection was performed. The transfection solution was prepared in advance according to the manufacturer's protocol. Plasmid $(5 \mu \mathrm{g})$ was added into $500 \mu \mathrm{l}$ DMEM, mixed and incubated at room temperature for 5 min. Lipofectamine $2000(10 \mu \mathrm{l})$ was added into DMEM, mixed and incubated at room temperature for $5 \mathrm{~min}$. The two solutions were subsequently mixed and incubated at room temperature for $20 \mathrm{~min}$. This mixture was added into 6-well plates, mixed and incubated. Complete DMEM was replaced $12 \mathrm{~h}$ after transfection and cultured in a constant temperature incubator at $37^{\circ} \mathrm{C}$ and $5 \% \mathrm{CO}_{2}$ for further experiments.
miR-34a expression detection. Total miRNA was extracted from cultivated HCT116 cells with an miRNA Extraction kit and the extracted miRNA was reverse-transcribed to cDNA with the TaqMan MicroRNA Reverse Transcription (RT) kit under the following reaction conditions: $16^{\circ} \mathrm{C}$ for $30 \mathrm{~min}, 42^{\circ} \mathrm{C}$ for $30 \mathrm{~min}$ and $85^{\circ} \mathrm{C}$ for $5 \mathrm{~min}$. Quantitative polymerase chain reaction (qPCR) was performed on the prepared cDNA using the TaqMan MicroRNA Detection kit under the following conditions: $95^{\circ} \mathrm{C}$ for $10 \mathrm{~min}$, followed by 40 cycles of $95^{\circ} \mathrm{C}$ for $15 \mathrm{sec}$ and $60^{\circ} \mathrm{C}$ for $1 \mathrm{~min}$. Following completion of qPCR, gene amplification was analyzed on a LightCycler ${ }^{\circledR} 480$ (Roche Diagnostics, Basel, Switzerland) to obtain the corresponding quantitation cycle $\left(\mathrm{C}_{\mathrm{q}}\right)$ value. U6 served as the internal reference to correct the copy number of the PCR template. Primer sequences were as follows: U6 forward, 5'-CTCGCTTCGGC AGCACA-3' and reverse, 5'-AACGCTTCACGAATTTGCGT-3'; miR-143a forward, 5'-GAGCTACAGTGCTTCATCTCAGCT CAGCA-3' and reverse, 5'-GCTGAGCTGAGATGAAGCACT GTAGCTCA-3'. Each group was tested in triplicate. Relative gene expression was calculated using the $2^{-\Delta \Delta \mathrm{Cq}}$ method (12).

Western blot analysis. HCT116 cells of the miR-34a transfection, negative control and blank control groups were cultured for $24 \mathrm{~h}$, following which the medium was removed. Precooled PBS was added to wash cells, followed by $10 \mu \mathrm{l}$ radioimmunoprecipitation assay lysis buffer (Shanghai Qiaoyu Industrial Co., Ltd., Shanghai, China). Following $10 \mathrm{~min}$, total protein was extracted and the protein concentration was quantified using the bicinchoninic acid method. Proteins $(50 \mu \mathrm{g})$ from each group were subjected to $10 \%$ SDS-PAGE. Following electrophoresis, proteins were transferred to a polyvinylidene difluoride membrane, which was blocked with $5 \%$ milk in TBS for $1 \mathrm{~h}$. Membranes were then incubated with the following primary antibodies at $4^{\circ} \mathrm{C}$ overnight: anti-Bcl-2 (cat. no. AB112; dilution, 1:1,000), anti-Bax (cat. no. YK1165; dilution, 1:500), anti-MMP-2 (cat. no. PA129183; dilution, 1:1,000), anti-MMP-9 (cat. no. 3852S; dilution, 1:1,000) and anti-GAPDH (cat. no. XB-1572; dilution, 1:1,000). The following day, the membrane was washed with TBS containing Tween-20 (0.05\%), and incubated with a horseradish peroxidase-conjugated IgG secondary antibody (cat. no. KC-GT-035; dilution, 1:5,000) for $1 \mathrm{~h}$ at room temperature. Protein bands were visualized with 3,3-diaminobenzidine, and BandScan 5.0 (Informer Technologies, Inc., Los Angeles, CA, USA) was used for analysis of the protein bands.

MTT assay for cell proliferation. HCT116 cells from the miR-34a transfection, negative control and blank control groups were seeded into a 96-well plate at a density of $10^{5} / \mathrm{ml}$, and $200 \mu \mathrm{l}$ complete DMEM was added. Following transfection, the cells were incubated at $37^{\circ} \mathrm{C}$ and $5 \% \mathrm{CO}_{2}$ for 24,36 , 48 or $72 \mathrm{~h}$. The medium was removed and MTT was added at $10 \mu \mathrm{l} /$ well. The cells were subsequently incubated at $37^{\circ} \mathrm{C}$ for $1 \mathrm{~h}$. A microplate reader (Thermo Fisher Scientific, Inc.) was used to record the absorbance of each well at $450 \mathrm{~nm}$, to calculate the inhibition of cell proliferation.

Transwell invasion assay. HCT116 cells from the miR-34a transfection, negative control and blank control groups were seeded into a 6-well plate. An 8- $\mu$ m diameter Corning culture 

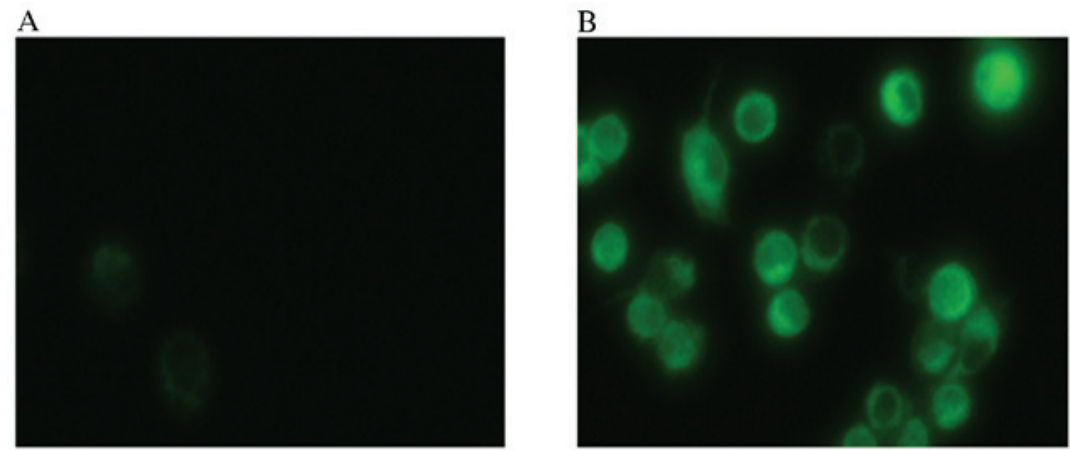

Figure 1. Confirmation of transfection of HCT116 cells with miR-34a. Fluorescence images of HCT116 cells in the (A) negative control and (B) miR-34a transfection groups reveal the presence of green fluorescent protein in the miR-34a-transfected cells, indicating successful transfection (magnification, $\mathrm{x} 40$ ). miR, microRNA.

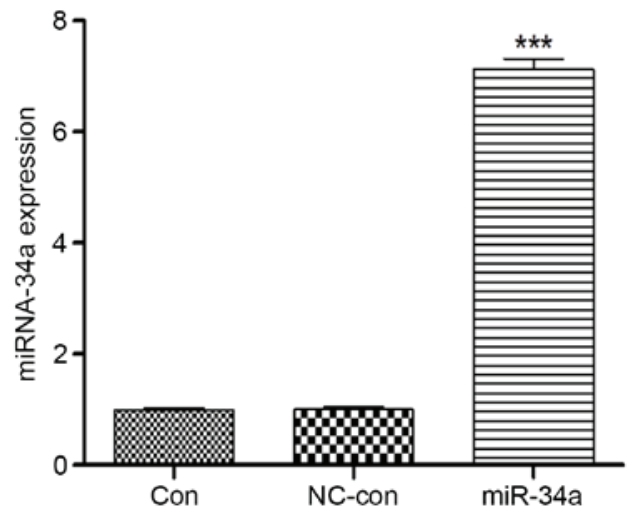

Figure 2. miR-34a expression levels in HCT116 cells following transfection. miR-341 expression levels in the blank control, negative control and miR-34a-transfected groups were determined by reverse transcription-quantitative polymerase chain reaction. Data are expressed as the mean \pm standard error $(n=3)$. ${ }^{* * *} \mathrm{P}<0.001$ vs. the Con group. miR, microRNA; Con, blank control; NC-con, negative control.

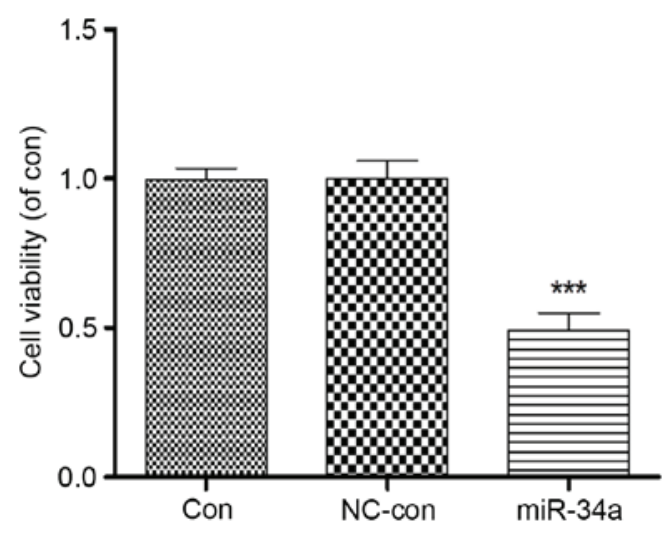

Figure 3. Viability of HCT116 cells following transfection. Cell viability in the blank control, negative control and miR-34a-transfected groups were determined by an MTT assay. Data are expressed as the mean \pm standard error $(n=3)$. ${ }^{* * *} \mathrm{P}<0.001$ vs. the Con group. miR, microRNA; Con, blank control; NC-con, negative control.

chamber was used to assess the invasiveness of HCT116 cells. The chamber membrane was coated with a single layer of Matrigel in advance. HCT116 cells $\left(10^{5}\right)$ from the transfection, negative control and blank control groups were added to the upper chamber, whereas complete DMEM was added to the lower chamber, and plates were incubated $37^{\circ} \mathrm{C}$ for $24 \mathrm{~h}$. The culture medium was removed, and cells were fixed with $4 \%$ paraformaldehyde and stained with hematoxylin. A total of five fields of view were randomly selected under a light microscope to count cells. Cell invasion rate $=$ (number of cells penetrating the lower chamber/number of cells penetrating the lower chamber in the blank control group) x 100 .

Statistical analysis. Data analysis was performed using GraphPad Prism software version 5.0 (GraphPad Software, Inc., La Jolla, CA, USA). The data are expressed as the mean \pm standard error. A one-way analysis of variance was performed to compare groups followed by a Dunnett's test. $\mathrm{P}<0.05$ was considered to indicate a statistically significant difference.

\section{Results}

Upregulation of miR-34a expression levels in miR-34a-transfected HCT116 cells. A total of $24 \mathrm{~h}$ after transfection of HCT116 cells with miR-34, visible fluorescence was detected from the GFP present in the plasmid (Fig. 1), which suggested a successful transfection. Total miRNA was extracted from the cells for RT-qPCR, which demonstrated that miR-34a expression levels in HCT116 cells increased 7.46 \pm 1.36 -fold compared with the blank control group $(\mathrm{P}<0.001)$, whereas no significant differences were identified between the negative control $(0.99 \pm 0.01)$ and blank control $(1.00 \pm 0.02)$ groups (Fig. 2). Therefore, transfection of HCT116 cells with miR-34a significantly increased the expression levels of miR-34a.

miR-34a transfection inhibits HCT116 cell proliferation. Proliferation of HCT116 cells was analyzed following transfection with miR-34a. An MTT assay revealed that proliferation of HCT116 cells overexpressing miR-34a was significantly inhibited to $0.49 \pm 0.11$ compared with the blank control group $(\mathrm{P}<0.001)$, whereas no significant differences were identified between the negative control $(1.00 \pm 0.08)$ and blank control $(0.99 \pm 0.06)$ groups (Fig. 3). This suggested a close association between miR-34a and the proliferation of HCT116 cells.

miR-34a transfection influences the protein expression levels of B-cell lymphoma $2(B c l-2)$ and $B c l$-2-associated $X$ protein $(B A X)$. Bcl-2 and BAX are important molecules in 


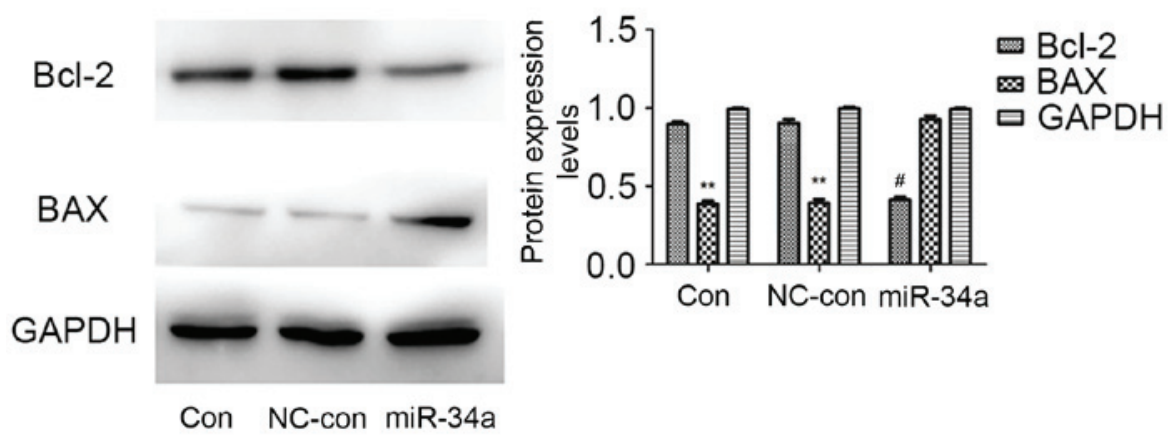

Figure 4. Protein expression levels of Bcl-2 and BAX in HCT116 cells following transfection. Protein expression levels in the blank control, negative control and miRNA-34a-transfected groups were determined by western blotting, with GAPDH serving as the internal reference. Data are presented as the mean \pm standard error $(\mathrm{n}=3)$. ${ }^{*} \mathrm{P}<0.05$ vs. the Con group; ${ }^{* *} \mathrm{P}<0.01$ vs. the miR-34a group. Bcl-2, B-cell lymphoma 2; BAX, B-cell lymphoma 2-associated X protein; miR, microRNA; Con, blank control; NC-con, negative control.

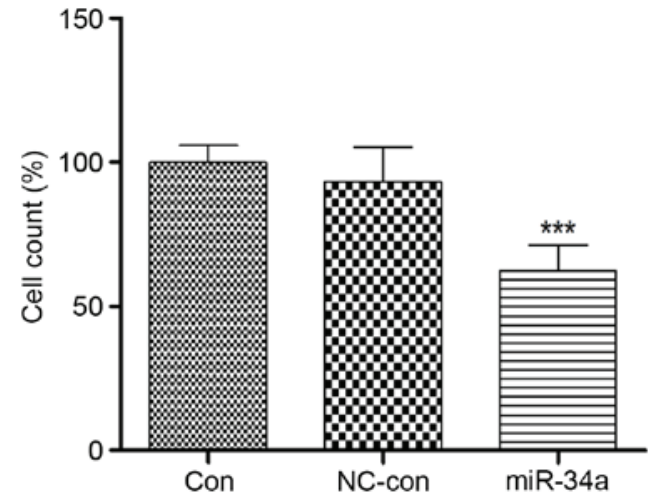

Figure 5. Invasiveness of HCT116 cells following transfection. Invasiveness in the blank control, negative control and miR-34a-transfected groups was determined by a Transwell assay. Data are expressed as the mean \pm standard error $(\mathrm{n}=3){ }^{* * * *} \mathrm{P}<0.001$ vs. the Con group. miR, microRNA; Con, blank control; NC-con, negative control.

apoptosis. Western blotting revealed that, compared with the blank control and negative control groups, the protein expression levels of Bcl-2 were markedly reduced in the miR-34a transfected group $(\mathrm{P}=0.002)$, whereas those of $\mathrm{BAX}$ were significantly increased $(\mathrm{P}=0.010)$. No significant differences were identified between the blank control and negative control groups (Fig. 4). This suggested that miR-34a may inhibit the proliferation of HCT116 cells by regulating the protein expression levels of Bcl-2 and BAX.

miR-34a transfection influences the invasiveness of HCT116 cells. Colon cancer is refractory primarily due to its ability to invade and metastasize. Therefore, the present study assessed the invasiveness of miR-34a-transfected HCT116 cells. A Transwell assay demonstrated that the invasiveness of HCT116 cells overexpressing miR-34a was significantly inhibited, to $62.76 \pm 8.44 \%$ of the level in the blank control group $(\mathrm{P}<0.001)$. No significant differences were identified between the negative control $(92.98 \pm 10.13 \%)$ and blank control $(100.51 \pm 8.22 \%)$ groups (Fig. 5). This suggested that miR-34a may be involved in HCT116 cell invasion.

miR-34a transfection influences the protein expression levels of matrix metalloproteinase (MMP)-2 and -9. There is a close association between the activity of MMPs and the invasion and metastasis of colon cancer cells. Western blot analysis demonstrated that compared with the blank control and negative control groups, protein expression levels of MMP-2 and MMP-9 were markedly reduced in the miR-34a-transfected group, the MMP-9 protein expression levels to a greater extent. No significant differences were identified between the blank control and negative control groups (Fig. 6). This suggested that miR-34a may inhibit the invasion and metastasis of

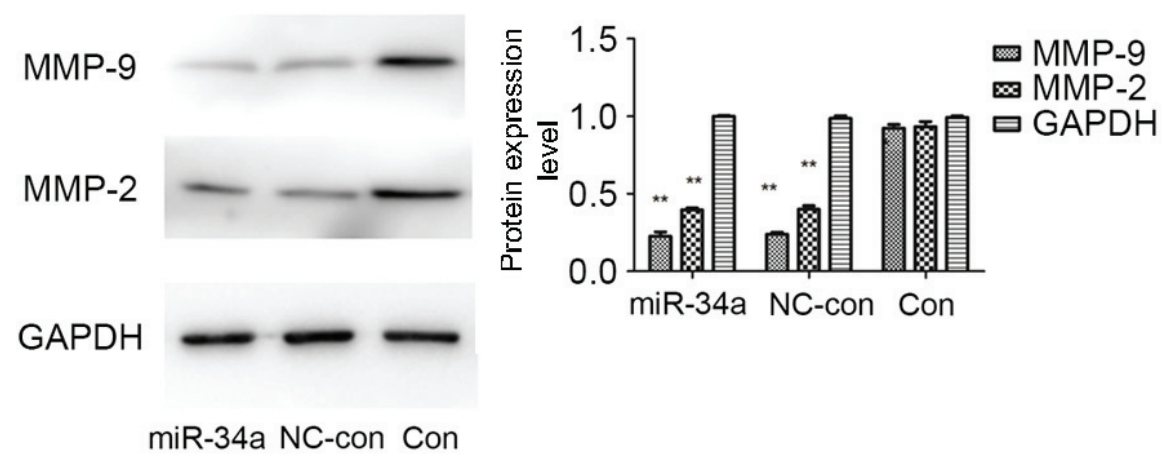

Figure 6. Protein expression levels of MMP-2 and MMP-9 in HCT116 cells following transfection with miRNA-34a. Protein expression levels in the blank control, negative control and miRNA-34a-transfected groups were determined by western blotting, with GAPDH serving as the internal reference. Data are expressed as the mean \pm standard error $(\mathrm{n}=3)$. ${ }^{*} \mathrm{P}<0.05$ and ${ }^{* *} \mathrm{P}<0.01$ vs. the Con group. MMP, matrix metalloproteinase; miRNA-34a, microRNA-34a; Con, blank control; NC-con, negative control. 
HCT116 cells by regulating the protein expression levels of MMP-2 and MMP-9.

\section{Discussion}

Cancer of the colon is a common malignant digestive tract tumor in China, and up to 8 million new cases are diagnosed every year worldwide. Characteristics of the disease include invasive growth, a high degree of malignancy and a high recurrence rate. Its etiology and underlying mechanisms remain to be understood, providing challenges for the clinical treatment of the disease $(13,14)$. Further investigation of the underlying molecular mechanisms and drug targets of colon cancer is therefore required. miRNAs are single-stranded, endogenous, non-coding small molecule RNAs, the functions of which have become clearer in recent years. It is highly conserved in structure, and may alter this by specific binding to the target mRNA at the 3'-untranslated region, promoting the degradation of the target gene mRNA or inhibiting its translation and transcription, and therefore regulating target protein expression (15-17). Previous studies have demonstrated that numerous miRNAs are located in chromosomal regions of the tumor-associated genomes, which possess different functions and serve different regulatory roles in the malignant biological behaviors of tumor cells, particularly proliferation, invasion and metastasis (1). Functional studies on miRNA provide novel insights and strategies for the diagnosis and treatment of a variety of tumors, including colon cancer. Such studies have increased or decreased the expression levels of a specific miRNA, via expression vectors or antisense miRNA, to inhibit the growth and metastasis of tumor cells (16). In addition, previous studies have demonstrated the differential expression of multiple miRNAs in colon cancer. For example, Wang et al (18) demonstrated that miRNA-21 inhibited the proliferation and cell cycles of colon cancer cells by targeted regulation of CDC25a expression. In addition, miR-143 and miR-145 have been implicated in the proliferation and chemosensitivity of colon cancer cells, thus rendering them potential diagnostic and therapeutic targets $(19,20)$. miR-34a may promote the apoptosis of glioma cells, block the cell cycle at G1 phase and inhibit proliferation; in addition, it may inhibit the invasion and metastasis of tumor cells (21). Furthermore, miR-34a reveals different regulatory functions in a variety of cancers, including prostate, liver and lung. Therefore, it is considered an important tumor suppressor (22). However, a limited number of studies have been performed on the role of miR-34a in colon cancer.

To investigate the role of miR-34a in colon cancer, the present study designed and synthesized a eukaryotic expression vector of miR-34a and transfected it into HCT116 cells. RT-qPCR demonstrated a significant increase of miR-34a expression levels in transfected cells. Overexpression of miR-34a inhibited the proliferation, invasion and metastasis of HCT116 cells. The results demonstrated that miR-34a inhibited tumor-associated genes in HCT116 cells, which indicated that miR-34a may be used as a potential target for gene therapy of colon cancer, consistent with the findings of previous studies $(4,19)$. Certain studies have identified that certain miRNAs regulate target proteins associated with the proliferation, invasion and metastasis of cancer cells; for example, miR-146b may inhibit the invasion of glioma cells by regulating MMP expression levels (23). Therefore, the present study examined the expression of proteins closely associated with cell proliferation and metastasis, and the results were consistent with those of previous studies. Protein expression levels of Bcl-2 were suppressed, whereas those of BAX were upregulated following miR-34a overexpression. Protein levels of both MMP-2 and MMP-9, which are associated with tumor metastasis, were inhibited. This suggested that miR-34a may reduce colon cancer cell proliferation and invasion by regulating the protein expression levels of Bcl-2/BAX, and MMP-2 and MMP-9.

In conclusion, the results of the present study demonstrated that overexpression of miR-34a may inhibit the proliferation, invasion and metastasis of HCT116 cells. This effect may be associated with the regulation of protein expression levels of Bcl-2/BAX, and MMP-2 and MMP-9. These findings suggested that miR-34a may be a potential target for the diagnosis and treatment of colon cancer, and provides a theoretical and experimental basis for subsequent studies.

\section{Acknowledgements}

The present study was supported by the Joint Funds of the Natural Science Foundation of Liaoning Province (grant nos. 2013023024 and 2015020324), the National Natural Science Foundation of China (grant no. 81172052) and the Yingcai Program of Dalian Medical University.

\section{References}

1. Adamowicz K and Zaucha R: Evaluation of the impact of cancer treatment on the adoption and consolidation of pro-health attitudes in the field of cancer in treated patients with colon cancer. J Cancer Educ 2016 (Epub ahead of print).

2. Kotelevets L, Chastre E, Desmaële D and Couvreur P: Nanotechnologies for the treatment of colon cancer: From old drugs to new hope. Int J Pharm 514: 24-40, 2016.

3. Watanabe T, Itabashi M, Shimada Y, Tanaka S, Ito Y, Ajioka Y, Hamaguchi T, Hyodo I, Igarashi M, Ishida $\mathrm{H}$, et al: Japanese Society for Cancer of the Colon and Rectum (JSCCR) guidelines 2010 for the treatment of colorectal cancer. Int J Clin Oncol 17: $1-29,2012$.

4. Krol J, Loedige I and Filipowicz W: The widespread regulation of microRNA biogenesis, function and decay. Nat Rev Gene 11: 597-610, 2010.

5. Fabian MR, Sonenberg N and Filipowicz W: Regulation of mRNA translation and stability by microRNAs. Annu Rev Biochem 79: 351-379, 2010.

6. Flynt AS and Lai EC: Biological principles of microRNA-mediated regulation: Shared themes amid diversity. Nat Rev Genet 9: 831-842, 2008.

7. Ventura A and Jacks T: MicroRNAs and cancer: Short RNAs go a long way. Cell 136: 586-591, 2009.

8. Calin GA, Liu CG, Sevignani C, Ferracin M, Felli N, Dumitru CD, Shimizu M, Cimmino A, Zupo S, Dono M, et al: MicroRNA profiling reveals distinct signatures in B cell chronic lymphocytic leukemias. Proc Natl Acad Sci USA 101: 11755-11760, 2004.

9. Kastl L, Brown I and Schofield AC: miRNA-34a is associated with docetaxel resistance in human breast cancer cells. Breast Cancer Res Treat 131: 445-454, 2012.

10. Rathod SS, Rani SB, Khan M, Muzumdar D and Shiras A: Tumor suppressive miRNA-34a suppresses cell proliferation and tumor growth of glioma stem cells by targeting Akt and Wnt signaling pathways. FEBS Open Bio 4: 485-495, 2014.

11. Rodriguez-Ubreva J, Ciudad L, van Oevelen C, Parra M and Graf T: C/EBPa-mediated activation of microRNAs 34a and 223 inhibits Lef1 expression to achieve efficient reprogramming into macrophages. Mol Cell Biol 34: 1145-1157, 2014. 
12. Livak KJ and Schmittgen TD: Analysis of relative gene expression data using real-time quantitative PCR and the 2(-Delta Delta C(T)) Method. Methods 25: 402-408, 2001.

13. Yothers G, O'Connell MJ, Allegra CJ, Kuebler JP, Colangelo LH, Petrelli NJ and Wolmark N: Oxaliplatin as adjuvant therapy for colon cancer: Updated results of NSABP C-07 trial, including survival and subset analyses. J Clin Oncol 29: 3768-3774, 2011.

14. Volinia S, Calin GA, Liu CG, Ambs S, Cimmino A, Petrocca F, Visone R, Iorio M, Roldo C, Ferracin M, et al: A microRNA expression signature of human solid tumors defines cancer gene targets. Proc Natl Acad Sci USA 103: 2257-2261, 2006.

15. Plönes T, Elze M, Kayser G, Pfeifer D, Burger M and Zissel G: mRNA and miRNA analyses in cytologically positive endobronchial ultrasound-guided transbronchial needle aspiration: Implications for molecular staging in lung cancer patients. Cancer Cytopathol 122: 292-298, 2014.

16. Alexandrov PN, Zhao Y, Jones BM, Bhattacharjee S and Lukiw WJ: Expression of the phagocytosis-essential protein TREM2 is down-regulated by an aluminum-induced miRNA-34a in a murine microglial cell line. J Inorg Biochem 128: 267-269, 2013.

17. Castro RE, Ferreira DM,Afonso MB, Borralho PM,Machado MV, Cortez-Pinto H and Rodrigues CM: miR-34a/SIRT1/p53 is suppressed by ursodeoxycholic acid in the rat liver and activated by disease severity in human non-alcoholic fatty liver disease. J Hepatol 58: 119-125, 2013.
18. Wang P, Zou F, Zhang X, Li H, Dulak A, Tomko RJ Jr, Lazo JS, Wang Z, Zhang L and Yu J: microRNA-21 negatively regulates Cdc25A and cell cycle progression in colon cancer cells. Cancer Res 69: 8157-8165, 2009.

19. Sun R, Chen P, Li L, Sun H, Nie X, Liang Y, Yuan F, Pu Y, Bai P, Zhang L and Gao L: A polymorphism rs4705341 in the flanking region of miR-143/145 predicts risk and prognosis of colorectal cancer. Oncotarget 2016 (Epub ahead of print).

20. Wei YS, Xiang Y, Liao PH, Wang JL and Peng YF: An rs 4705342 $\mathrm{T}>\mathrm{C}$ polymorphism in the promoter of miR-143/145 is associated with a decreased risk of ischemic stroke. Sci Rep 6: 34620, 2016.

21. Li N, Fu H, Tie Y, Hu Z, Kong W, Wu Y and Zheng X: miR-34a inhibits migration and invasion by down-regulation of c-Met expression in human hepatocellular carcinoma cells. Cancer Lett 275: 44-53, 2009.

22. Gallardo E, Navarro A, Viñolas N, Marrades RM, Diaz T, Gel B, Quera A, Bandres E, Garcia-Foncillas J, Ramirez J and Monzo M: miR-34a as a prognostic marker of relapse in surgically resected non-small-cell lung cancer. Carcinogenesis 30: 1903-1909, 2009.

23. Tazawa $H$, Tsuchiya $N$, Izumiya $M$ and Nakagama $H$ : Tumor-suppressive miR-34a induces senescence-like growth arrest through modulation of the E2F pathway in human colon cancer cells. Proc Natl Acad Sci USA 104: 15472-15477, 2007. 